\title{
Stirling Cycle Solar Power System Design and Simulation Quan-Run DONG ${ }^{\mathrm{a}}$ and Yong-Qiang $\mathrm{SHI}^{\mathrm{b}, *}$
}

\author{
School of Power and Energy, Northwestern Polytechnical University, Xi'an, China, 710129
}

adongquanrun@mail.nwpu.edu.cn, byqshi@nwpu.edu.cn

Keywords: Stirling cycle, solar power generation, scenery integration, system design, simulation calculation.

\begin{abstract}
A Stirling cycle solar wind combined power generation system was designed, and a theoretical analysis model of the power and efficiency for each subsystem of the system was theoretically constructed. Firstly, the Stirling machine and the solar cooker are used to focus on the solar energy. The vertical fan is used instead of the traditional fan. The combination of the overrunning clutch and the drum-shaped gear coupling realizes the combination of wind energy and light energy to complete the design of the power generation system. Then, a theoretical analysis model such as the output power of the Stirling machine, the generator power of the shaft, and the overall efficiency of the generator is established. The theoretical analysis model can effectively describe the various functions and efficiency analysis of the actual Stirling cycle solar wind combined power generation system.
\end{abstract}

\section{Introduction}

The development of renewable, clean and environmentally friendly green energy power generation methods that can replace one-time energy has become a major development trend in power development. The clean and pollution-free power generation methods such as solar energy and wind energy have received increasing attention from countries. There are mainly two types of solar power generation: one is photovoltaic power generation, and the other is solar thermal power generation. The rapid development of photovoltaic power generation technology has shown good power generation efficiency in practical applications, but when producing silicon photovoltaic panels for photovoltaic power generation, it needs to consume a lot of energy, and it also generates a lot of pollution. The use of solar energy as the heat source of Stirling cycle power generation does not require the use of large-scale, high-energy consumption single-crystal silicon, polysilicon photovoltaic panels; give full play to the characteristics of solar energy clean. Therefore, designing and optimizing a solar energy system based on the Stirling cycle has important practical engineering application significance.

The innovation of this article mainly focuses on the following two aspects:

1) A solar energy combined wind power generation system based on the Stirling cycle is designed. The vertical axis fan replaces the wind turbine power generator; the cooperation of various devices such as overrunning clutches, couplings, solar cookers and regenerators is adopted. To achieve solar energy and wind energy cogeneration, effectively improving the efficiency of the use of solar energy.

2) Starting with the theoretical analysis, the theoretical analysis model of the output power of the Stirling machine, the work efficiency of the shaft, the efficiency of the shaft blade and the overall efficiency of the generator are established. The physical model of the model was established to verify the correctness and applicability of the model.

\section{Design Plan}

The Stirling cycle is a closed and generalized Carnot cycle with the most thermodynamically theoretically optimal heat recovery at a constant volume. It was named after the British engineer Robert Stirling (1790-1878) was first proposed in 1816. The Stirling cycle is a reversible cycle consisting of two constant volume endothermic processes and two constant temperature expansion 
processes, and the heat released during the constant volume and heat release process is exactly absorbed by the constant volume and endothermic process. The heat engine absorbs heat from the high temperature heat source during constant temperature expansion, and releases heat to the low temperature heat source during the constant temperature compression process.

\section{Stirling Cycle Solar Wind Energy Combined Power System Design}

Solar power systems based on the Stirling cycle include solar cookers, Stirling machines, flywheels, drum gear couplings, overrunning clutches, vertical shaft fans, and generators. The model is shown in Figure 1. The overall structure of a solar power system based on the Stirling cycle is shown in Fig. 2.

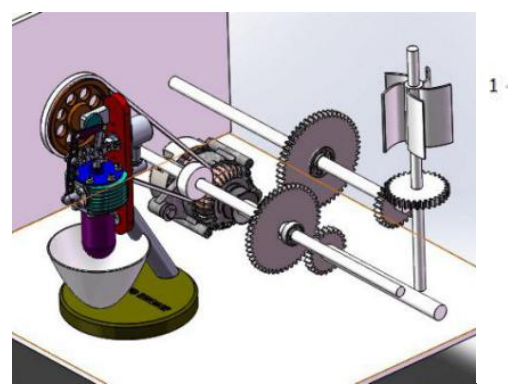

Fig. 1 Stirling generator model

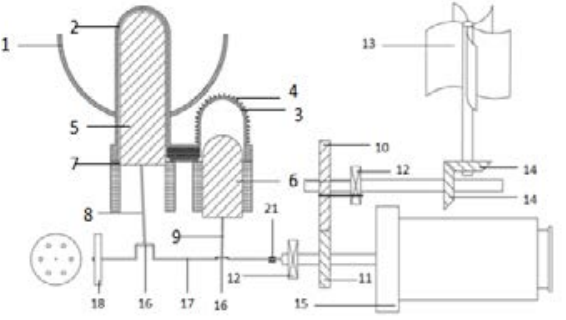

Fig. 2 Overall structure

The main part numbers and names in the figure are shown in the following table:

Table 1 Description of each part number of Sterling power generation system

\begin{tabular}{cccc}
\hline Number & Name & Number & Name \\
\hline 1 & Solar Cooker & 12 & Overrunning clutch \\
2 & Hot cylinder & 13 & Vertical fan \\
3 & Cold cylinder & 14 & Bevel gear \\
4 & heat sink & 15 & generator \\
5 & Hot cylinder piston & 16 & Sleeve \\
6 & Cold cylinder piston & 17 & Crankshaft \\
7 & Reheater & 18 & flywheel \\
8 & Hot cylinder connecting & 19 & Shoulder \\
& rod & & Top nut \\
9 & Cold cylinder connecting & 20 & \\
& rod & 21 & Drum gear coupling \\
10 & big gear & & \\
11 & gear & &
\end{tabular}

The solar cooker is a hemispherical concave mirror mounted on a hot air cylinder of a Stirling machine. The condenser heats the hot air cylinder of the Stirling machine to provide heat.

The Stirling machine is an external combustion engine focusing on sunlight as a heat source. The main structures include a hot air cylinder, a cold air cylinder, a piston, a heat sink, a regenerator, a crankshaft, a connecting rod, and a sleeve. The heat sink is a concentric annular metal foil located on a cold cylinder for enhancing the cooling efficiency of the cold cylinder. The regenerator is located in the channel that connects the hot and cold cylinders. The crankshaft of the inner ring of the sleeve is steel and the bearing bush is brass. The cooperation of the two can reduce friction and increase the service life of the parts.

The flywheel is a six-hole steel wheel with a large mass that allows the crankshaft to run through the dead point when the crankshaft is running to top and bottom dead center. The angular energy can be released and stored to make the Stirling machine run more smoothly.

The drum-shaped gear coupling eliminates the internal forces caused by the different axes of the coupling front and rear axles. 
Overrunning clutches, also known as ratchets, act to transmit directional torque, which allows two shaft torques to be transmitted to one shaft to drive a generator to generate electricity.

The vertical axis fan is composed of five curved vertical axis fans, which can not only stably rotate at low wind speed, but also work stably under strong wind conditions, effectively widening the margin of available wind energy. The traditional mainstream wind turbines adopt the construction method of wind turbines and towers, and such tower wind turbines cannot work during strong winds. The vertical axis fan can generate electricity under various wind speed conditions, even against super typhoon, not easy to damage, and low in price.

\section{Workflow of Stirling Cycle Solar Power System}

1) Constant temperature compression: At the beginning of the cycle, the cold cylinder connecting rod 9 pushes the cold piston 6 upwards, the hot piston 5 does not move, the gas in the cold cylinder 3 is compressed, and heat is released outside the cylinder, and the gas temperature does not change.

2) Constant volume heating: the cold cylinder connecting rod 9 continues to push the cold piston 6 upwards, the hot piston 5 moves downwards under the control of the gas pressure and the hot cylinder connecting rod 8, and the gas in the cold cylinder 3 enters the heat through the regenerator 7 In the cylinder 2, the temperature rises as the gas passes through the regenerator 7 and the pressure rises.

3) Constant temperature expansion: The expansion of high temperature gas pushes the thermal piston 5 downwards to do work externally, and the heating source makes the temperature in the gas in the hot cylinder 2 constant during the expansion work, slow down the pressure drop, and the thermal piston 5 moves to the bottom dead point.

4) Constant volume cooling: The hot cylinder connecting rod 8 controls the hot piston 5 to move upwards, the cold piston 6 moves downwards, the gas releases heat as it passes through the regenerator 7, the temperature decreases, the pressure drops, and the hot and cold pistons move to their respective The end of the cycle is complete.

\section{Model Efficiency Analysis}

\section{Solar Stove Hot End Temperature Maintenance and Efficiency Calculation}

The hot-end temperature can be maintained by solar energy. The main solution is through the condenser.

The condenser can not only make up for the lack of light quantity and properly change the properties of the light emitted from the light source, but also focus the light on the object to be inspected in order to obtain the best focusing effect. The condenser can also provide energy for the hot end of the Stirling cycle. The structure is shown in Figure 3.

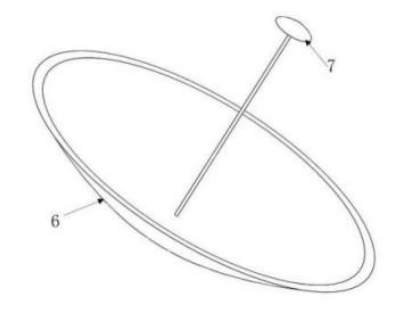

Fig. 3 condenser lens

(6--condenser, 7-receiver)

The condenser mirror reflects light onto the receiving mirror, where the condenser lens area is as large as possible and the receiving mirror area is as small as possible. Concentrators have errors in production and manufacturing that cause energy loss, and concentrator efficiency can be expressed by the concentrator cut-off efficiency: 


$$
\eta_{g}=1-e^{(-1 / 2 C \sigma)}
$$

Among them, $\sigma$ is the optical error of the condenser; $\mathrm{C}$ is the light gathering ratio, $C=A_{m} / A_{n}$; $A_{m}$ is the opening area of the condenser lens, $A_{n}$ is the opening area of the receiving mirror.

\section{Sterling Machine Output Work Analysis}

For the ideal Stirling cycle, the working fluid in the Stirling machine is evenly distributed and the ideal gas pressure formula is used. The total pressure of the Stirling machine is as follows:

Among them, $m$ is the mass of the working fluid, $R$ is the ideal gas constant, $V_{1}$ is the volume when the cold cylinder is compressed, $V_{2}$ is the volume of the cold cylinder, $V_{3}$ is the volume of the regenerative cavity, $V_{4}$ is the volume of the hot cylinder, $V_{5}$ is the expansion of the hot cylinder volume.

The work done on the drive crankshaft when the cold cylinder is compressed is as follows: (Let A be the rotation angle)

$$
W_{1}=\int P \frac{d V_{1}}{d \theta} d \theta
$$

The calculation formula for the work of driving the crankshaft when the hot cylinder is compressed is as follows:

$$
W_{2}=\int P \frac{d V_{5}}{d \theta} d \theta
$$

The total work is

$$
W=W_{1}+W_{2}
$$

\section{Shaft Power Generation Analysis}

The Stirling machine's shaft can drive a generator to drive. This article uses a simple stator rotor generator to generate electricity. The generator structure is shown in Figure 4.

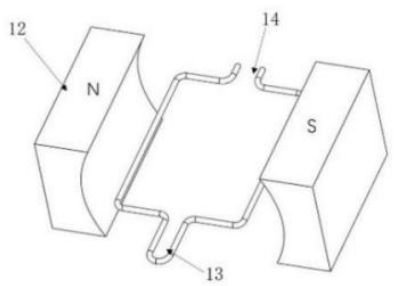

Fig. 4 Generator sketch

(12--permanent magnet, 13--rotary shaft access, 14-- load access)

Among them, the stator is a permanent magnet, the rotor is a wire frame, and the electromotive force is generated by cutting the magnetic induction wire. Electric power calculation formula is:

$$
W_{3}=\int_{0}^{t} \frac{U^{2}}{R} d t=\int_{0}^{t} \frac{(B l \omega r)^{2}}{R} d t
$$

Where B is the magnetic induction intensity, $l$ is the length of the wire that cuts the magnetic induction wire, $\mathrm{w}$ is the angular velocity of the wire frame, and $\mathrm{r}$ is the radius of the wire box. 


\section{Overall Efficiency of Solar Generators Based On the Stirling Cycle}

The overall efficiency of solar generators based on the Stirling cycle is the product of the efficiency of each part.

When using a collector, the total efficiency is calculated as:

$$
\eta_{2}=\eta_{1} \cdot \frac{W}{W_{3}}=\frac{c m \Delta T}{A_{s} G} \frac{\int P \frac{d V_{1}}{d \theta} d \theta+\int P \frac{d V_{5}}{d \theta} d \theta}{\int_{0}^{t} \frac{(B l \omega r)^{2}}{R} d t}
$$

When using a concentrator, the total efficiency is calculated as:

$$
\eta_{3}=\eta_{g} \cdot \frac{W}{W_{3}}=1-e^{(-1 / 2 C \sigma)} \frac{\int P \frac{d V_{1}}{d \theta} d \theta+\int P \frac{d V_{5}}{d \theta} d \theta}{\int_{0}^{t} \frac{(B \mid \omega r)^{2}}{R} d t}
$$

\section{Conclusion}

1) Analyzed the advantages of solar energy as a heat source for Stirling cycle power generation than traditional photovoltaic power generation methods. Stirling cycle solar power generation has higher power generation efficiency, low energy consumption and less pollution, and has good development prospects.

2) A Stirling cycle solar wind combined power generation system was designed to adapt to various wind speeds. Based on the solar concentrating design, the efficiency of solar energy utilization can be effectively improved, and electricity can be provided in areas with strong sunshine and high wind energy density. Energy has great market prospects.

3) The simulation calculation model of Stirling cycle power generation system was established. Through theoretical analysis, the formulas of various power and efficiency in Stirling cycle power generation system were deduced. The effectiveness of the system has certain reference value for the design of the Stirling cycle power generation system.

\section{References}

[1] Liu Xingyue, Wu Hongbin. Control Strategy and Operation Optimization of Combined Cooling, Heating and Power Systems for Solar Energy Utilization [J]. Automation of Electric Power Systems, 2015, 39(12):1-6.

[2] Zheng Tianqi, Ding Guozhong, Ou Changkai, et al. Volume matching of Stirling heat chamber expansion chamber and compression chamber [J]. Chinese Journal of Solar Energy, 2016, 37(4): 940-944.

[3]Singh N, Gibbs B M, Kaushik S C. EFFECT OF SOLAR COLLECTOR DESIGN PARAMETERS ON THE OPERATION OF SOLAR STIRLING POWER SYSTEM[J]. International Journal of Energy Research, 2015, 21(2):195-200.

[4] Guarato A, Moura C M, Bernardes M S, et al. AN EXPERIMENTAL THERMAL ANALYSIS OF AN ENGINE BASED ON STIRLING CYCLE MODEL ALPHA[C]. Abcm International Congress of Mechanical Engineering. 2017.

[5] Ding Shengping, Wang Yongzheng, Lü Ruijie, et al. Performance Simulation of Dish Solar Thermal Power Generation System Based on Stirling Machine[J]. Journal of Shandong University(Engineering Science), 2014(4):64-69. 
[6] Zhou Yuqing. Discussion of the Stirling Cycle's Ideal and Actual Efficiency [J]. Physics Bulletin, 2015(2): 93-96. 